\title{
Probing nuclear cluster symmetries through the harmonic oscillator
}

\author{
Martin Freer ${ }^{1, a}$, Rhiann Canavan ${ }^{2}$, Thomas Marsh ${ }^{1}$, James Souter ${ }^{1}$ \\ ${ }^{1}$ School of Physics and Astronomy, University of Birmingham, Birmingham B15 2TT, UK \\ ${ }^{2}$ Department of Physics, University of Surrey, Guildford, Surrey GU2 7XH, UK
}

Received: 24 December 2020 / Accepted: 21 February 2021 / Published online: 22 March 2021

(C) The Author(s) 2021

Communicated by Nicolas Alamanos

\begin{abstract}
The present contribution explores the symmetries of the deformed harmonic oscillator for both prolate and oblate deformations. It demonstrates the emergence of clustering from the degeneracies of the deformed harmonic oscillator and the appearance of the cluster structures in the associated densities. The universality of molecular structures is presented, demonstrating that molecular-like exchange of protons and neutrons is encoded into the mean-field. The nature of oblate-like structures is also explored.
\end{abstract}

Keywords Nuclei $\cdot$ Clustering and molecular structures

\section{Introduction}

The structure of light nuclei has become an important testing ground for our evolving understanding of the nature of the strong interaction as it plays out in the nuclear context. There has been a resurgence of interest in pinning down the details of the interaction and the importance of low and high momentum components and the role of correlations [1]. Light nuclei provide a fascinating balance of the role and behaviour of the single-particle, manifest in the mean-field, and the condensation of cluster-like structures from the mean-field. These structures evolve from the symmetries contained within the mean-field but are then amplified by the enhanced binding energy of the $\alpha$-particle as a cluster and can result in rather pronounced $\alpha$-cluster structures [2,3].

This co-existence of single-particle/shell model and clusterlike characteristics has been explored at length using the deformed harmonic oscillator as a framework [2], and although the harmonic potential is not a perfect description of the nuclear potential does provide a good starting point to understand more sophisticated nuclear models which explic-

a e-mail: M.Freer@bham.ac.uk (corresponding author) itly account for every nucleon and use realistic nucleonnucleon interactions [1]. These $a b$ initio approaches are extremely powerful and as computational power has grown have become the tool to properly connect the nature of the strong force with nuclear properties leading to the prospect that average interactions, effective forces and the mean-field approach which has served the field well, may be superseded through ab initio methods.

This is an exciting prospect, but these complex calculations need to be grounded in the reality of experimental measurement, where precision measurements are required to test the accuracy, and there is also the need to be guided by more simple approaches in order to provide meaning and context to the results. This particular contribution explores some of the guiding principles and symmetries that emerge from the deformed harmonic oscillator that may be used to inform more complex calculations.

\section{Deformed harmonic oscillator}

The deformed harmonic oscillator has the well-known shelllike structure where at integer ratios of axial deformations the shell structure is enhanced, Fig. 1. The deformed harmonic oscillator has been used as the basis for understanding deformed nuclei [4], and the symmetries associated with shell closures have been described in terms of the SU(3) group [5]. This shell structure may be associated with stable deformed structures and, as illustrated by Rae [6], cluster partitions.

The total energy at each level for the Deformed Harmonic Oscillator, $\mathrm{DHO}$ is given by:

$E=\hbar \omega_{x} n_{x}+\hbar \omega_{y} n_{y}+\hbar \omega_{z} n_{z}+\frac{3}{2} \hbar \omega_{0}$ 


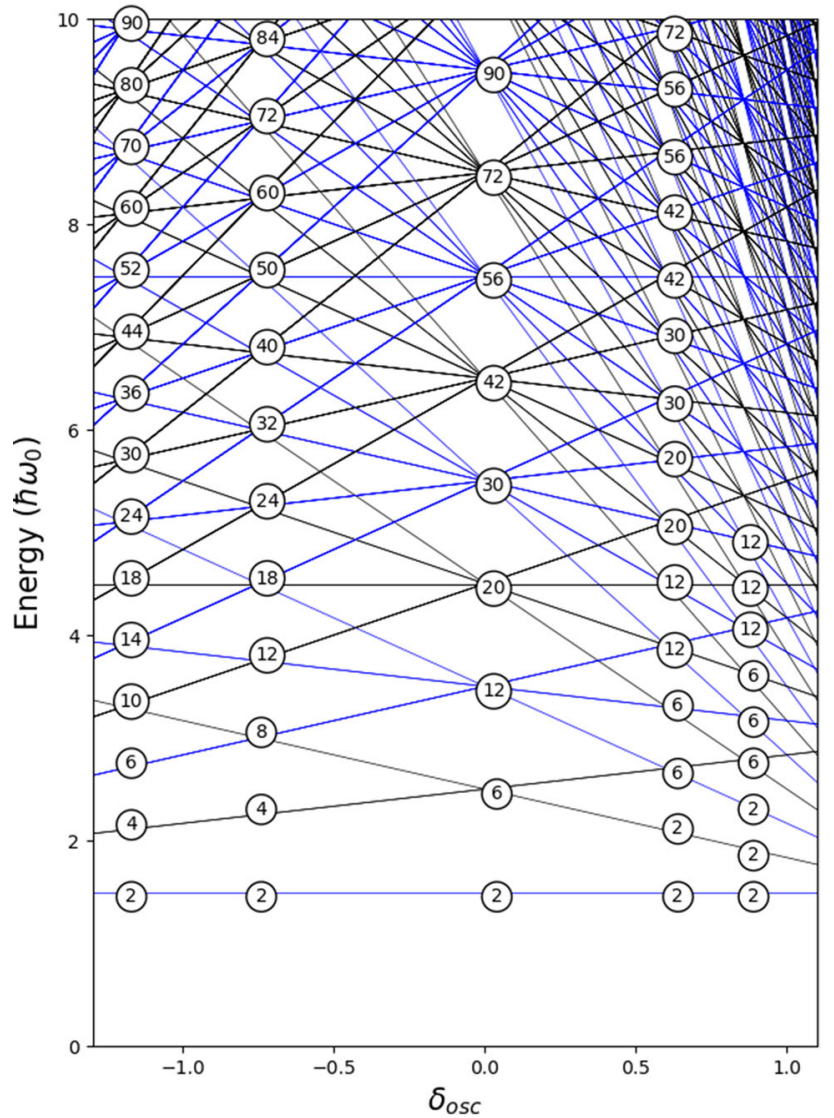

Fig. 1 The energy levels of the deformed harmonic oscillator as a function of the deformation parameter $\delta_{O S C}$. The labelling of the level crossings is in accordance with the degeneracy of the levels linked to the shell gaps. A degeneracy of 2 is equivalent to a single level which can accommodate either two-protons or two neutrons, spins anti-aligned

where $\omega_{x}, \omega_{y}$ and $\omega_{z}$ and $n_{x}, n_{y}$ and $n_{z}$ are the oscillation frequencies and number of oscillation quanta in the $x, y$, and $z$ directions respectively, and $\omega_{0}$ is the average oscillation frequency. The orbitals in the DHO are labelled by $\left[n_{x}, n_{y}\right.$, $\left.n_{z}\right]$ (or for axially symmetric nuclei $\left[n_{\perp}, n_{z}\right]$ where $n_{\perp}$ is the number of oscillator quanta perpendicular to the deformation axis), and the number of oscillator quanta in each Cartesian direction determines the orientation of that orbital. For a nucleus with, for example, a 2:1 deformation along the $z$-direction, the energy levels are given by:

$E=\frac{6}{5} \hbar \omega_{0} n_{x}+\frac{6}{5} \hbar \omega_{0} n_{y}+\frac{3}{5} \hbar \omega_{0} n_{z}+\frac{3}{2} \hbar \omega_{0}$.

This means that the $\left[n_{x}, n_{y}, n_{z}\right]=[1,0,0],[0,1,0]$ and $[0,0,2]$ levels become degenerate.

The association of cluster configurations with the shell structure $[6,7]$ follows from the degeneracies illustrated in Fig. 1. Within the DHO the nucleus ${ }^{8} \mathrm{Be}$, for example, is created through the population of the DHO orbitals, $\left[n_{x}, n_{y}\right.$, $\left.n_{z}\right]=[0,0,0]^{4}$ and $[0,0,1]^{4}$. At a 2:1 deformation this is seen
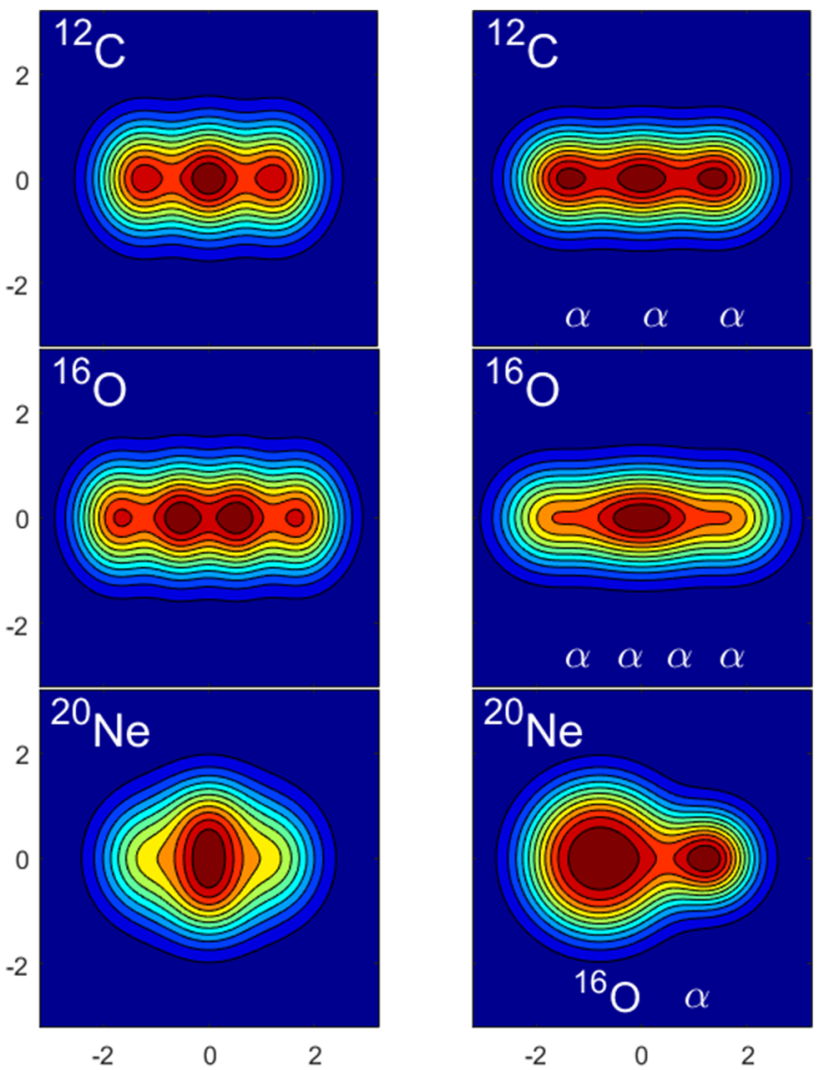

Fig. 2 (left) The DHO density calculations for the three nuclei ${ }^{12} \mathrm{C}$, ${ }^{16} \mathrm{O}$ and ${ }^{20} \mathrm{Ne}$ for the prolate deformations $3: 1,4: 1$ and 2:1, respectively. (right) The calculated densities for the three nuclei ${ }^{12} \mathrm{C},{ }^{16} \mathrm{O}$ and ${ }^{20} \mathrm{Ne}$ formed from superimposing the densities of the individual clusters, i.e. $3 \alpha, 4 \alpha$ and ${ }^{16} \mathrm{O}+\alpha$, respectively [8]

to be associated with the filling of the two orbitals each with $2 p+2 n$ with the spins of the particles coupled to $S=0$, i.e. $2 \alpha$-particles, and hence ${ }^{8} \mathrm{Be}$ may be thought of as $2 \alpha$ clusters. Remarkably, the densities calculated from this DHO configuration also reveal the two-centre cluster structure [7].

The next shell closure at a deformation of 2:1 occurs for ${ }^{20} \mathrm{Ne}$. Here the degeneracies are $2+(2+6)$ and may be connected with a ${ }^{4} \mathrm{He}+{ }^{16} \mathrm{O}$ cluster structure, as seen in Fig. 2 . The arrangements of the alpha particles may equally be visualised as $\alpha-{ }^{12} \mathrm{C}-\alpha$, with the plane of the ${ }^{12} \mathrm{C}$ nucleus aligned perpendicular to the deformation axis, or, as indicated, by ${ }^{16} \mathrm{O}-\alpha$ with a double tetrahedral structure. This recognises that the spherical ${ }^{16} \mathrm{O}$ nucleus can be considered a tetrahedral arrangement of $4 \alpha$-particles. The next shell closure at the 2:1 deformation occurs for degeneracies $2+2+8+8$ which would correspond to ${ }^{32} \mathrm{~S}$ with a ${ }^{16} \mathrm{O}+{ }^{16} \mathrm{O}$ cluster structure.

A similar situation occurs at a deformation of $3: 1$, with for example the shell gap at cumulative degeneracies of $2+2+2+6+6+6$ being linked to the $3: 1$ deformed state in ${ }^{48} \mathrm{Cr}$ and a ${ }^{16} \mathrm{O}+{ }^{16} \mathrm{O}+{ }^{16} \mathrm{O}$ cluster configuration [8], Fig. 3 .

Figures 2 and 3 illustrate the symmetries found in the deformed harmonic oscillator degeneracies also appear in 

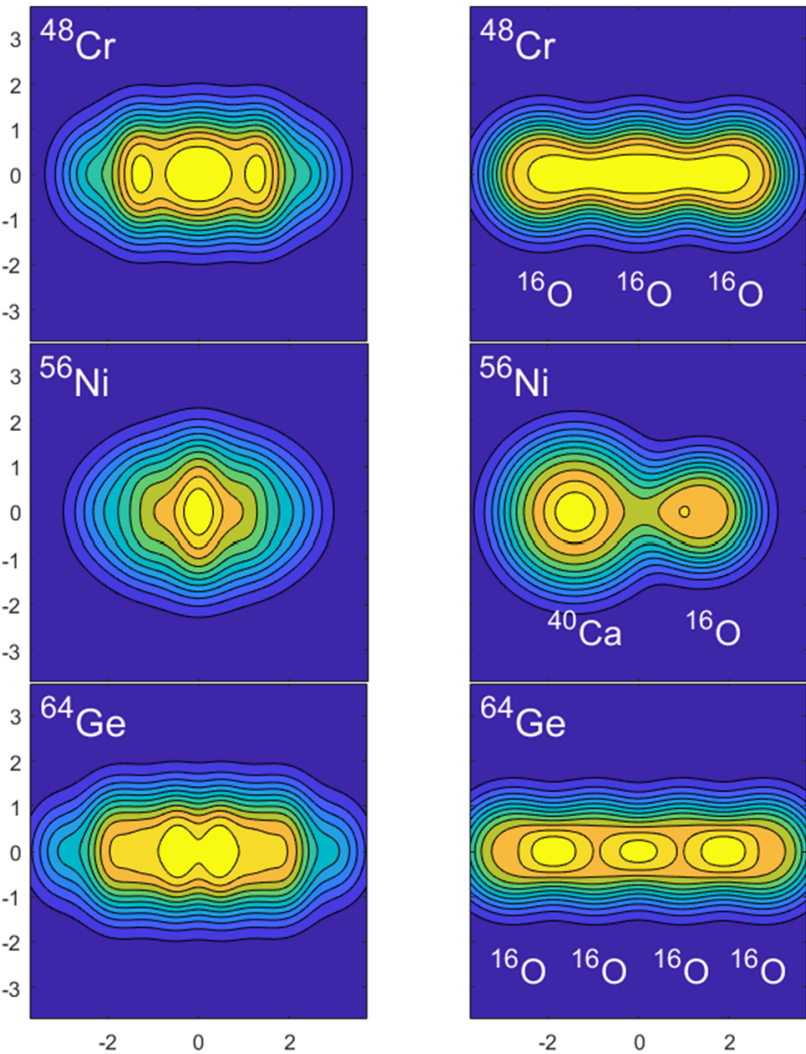

Fig. 3 (left) The DHO densities for the nuclei ${ }^{48} \mathrm{Cr},{ }^{56} \mathrm{Ni}$ and ${ }^{64} \mathrm{Ge}$, which reveal the underlying cluster structures ${ }^{16} \mathrm{O}+{ }^{16} \mathrm{O}+{ }^{16} \mathrm{O},{ }^{40} \mathrm{Ca}+{ }^{16} \mathrm{O}$ and ${ }^{16} \mathrm{O}+{ }^{16} \mathrm{O}+{ }^{16} \mathrm{O}+{ }^{16} \mathrm{O}$, respectively. (right) The densities of the three cluster structures ${ }^{16} \mathrm{O}+{ }^{16} \mathrm{O}+{ }^{16} \mathrm{O},{ }^{40} \mathrm{Ca}+{ }^{16} \mathrm{O}$ and ${ }^{16} \mathrm{O}+{ }^{16} \mathrm{O}+{ }^{16} \mathrm{O}+{ }^{16} \mathrm{O}$ created by superimposing the corresponding densities [8]

the harmonic oscillator densities. These figures show the harmonic oscillator on the left hand panel and the density that might result from summing the densities of the individual cluster components on the right hand side. It is interesting to note that, if anything, the cluster structure is more enhanced in the DHO densities than the aggregated cluster densities. This demonstrates that at the type of cluster separations that are predicted by the mean-field approach that the Pauli principle plays a defining role in the nature of the structure. The interesting feature is that these symmetries and structures do not wash out when the clusters become more complex and the nuclei are heavier as illustrated in the case of the linear arrangement of $4{ }^{16} \mathrm{O}$ clusters in the calculation of ${ }^{64} \mathrm{Ge}$ in Fig. 3. These structures illustrate that the symmetries are not only present in a trivial manner for light nuclear systems, but also have a role to play in heavier ones.

There is also copious evidence for $\alpha$-particle like structures in light nuclei [1,2], with two irresistible cases ${ }^{8} \mathrm{Be}$ and ${ }^{12} \mathrm{C}$, and most famously the Hoyle state in the latter, which we return to later.

However, for heavier systems, from an experimental perspective, their existence is harder to demonstrate as the struc-

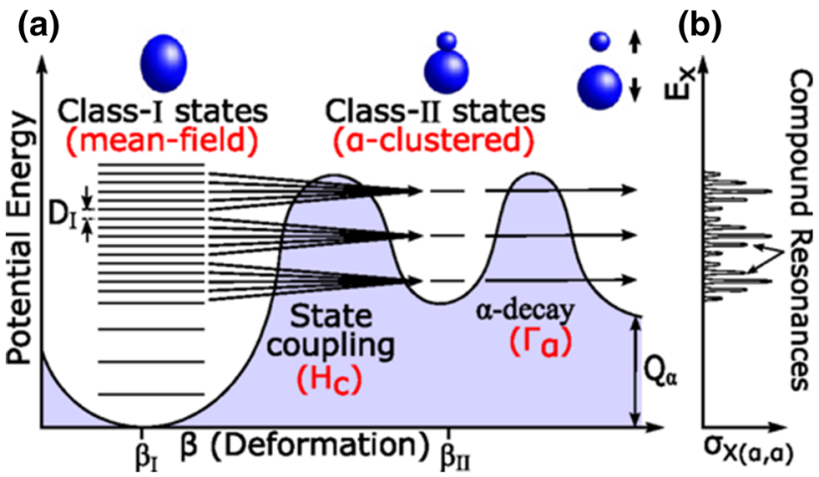

Fig. 4 A schematic of a double humped decay barrier. a The application of a double decay fission barrier to fragmented $\alpha$-clustered states with class-I (shell-model) and class-II (cluster) states indicated. b A schematic scattering cross section resulting from this structure [9]

tures exist at high excitation energy adjacent to the respective cluster decay threshold, the Ikeda picture [10], and at such energies are embedded in a highly dense sea of other states which fragments the states through coupling, resulting in a complex spectrum, Fig. 4. Such fragmented states are observed in the barrier resonances seen in systems such as ${ }^{12} \mathrm{C}+{ }^{12} \mathrm{C},{ }^{12} \mathrm{C}+{ }^{16} \mathrm{O}$ and ${ }^{16} \mathrm{O}+{ }^{16} \mathrm{O}[11]$.

\section{Oblate nuclei}

Much focus to-date has been on the prolate side of the DHO level scheme, with a range of cluster and molecular structures (see later) being revealed. The symmetries of the oblate, axially-symmetric, deformed harmonic oscillator are less obvious.

In the case of the prolate nuclei at 2:1 deformation the spherical degeneracies repeat sequentially, i.e. 2, 2, 6, 6, 12, $12, \ldots$ where each shell closure is associated with the next number in the sequence. For oblate nuclei the situation is different (Fig. 5), the pattern for a deformation of 1:2 is $2+0,2+2,6+2,6+6,12+6,12+12, \ldots$ At a prolate deformation of $3: 1$ the sequence is $2,2,2,6,6,6,12,12,12 \ldots$ and on the oblate side at $1: 32+0+0,2+2+0,2+2+2,6+2+2,6+6+6$, $12+6+6,12+12+6,12+12+12 \ldots$

As a starting point, and as explored by Zhang and Rae $[12,13]$, it is tempting to explore these planar-like structures starting with triangular arrangements of $\alpha$-particles. The triangular numbers are $1,3,6,10,15, \ldots$. , and in terms of the present scheme $\alpha$-particles arranged in planar triangles would be associated with degeneracies of $2,6,12,20,30, \ldots$. i.e. double the triangular numbers. Curiously, these are also the spherical degeneracies. This can be understood as spherical nuclei can be formed from the arrangement of layers of increasing triangular structures to form tetrahedral arrangements. In Ref. [13] there is a description of the assembly of 


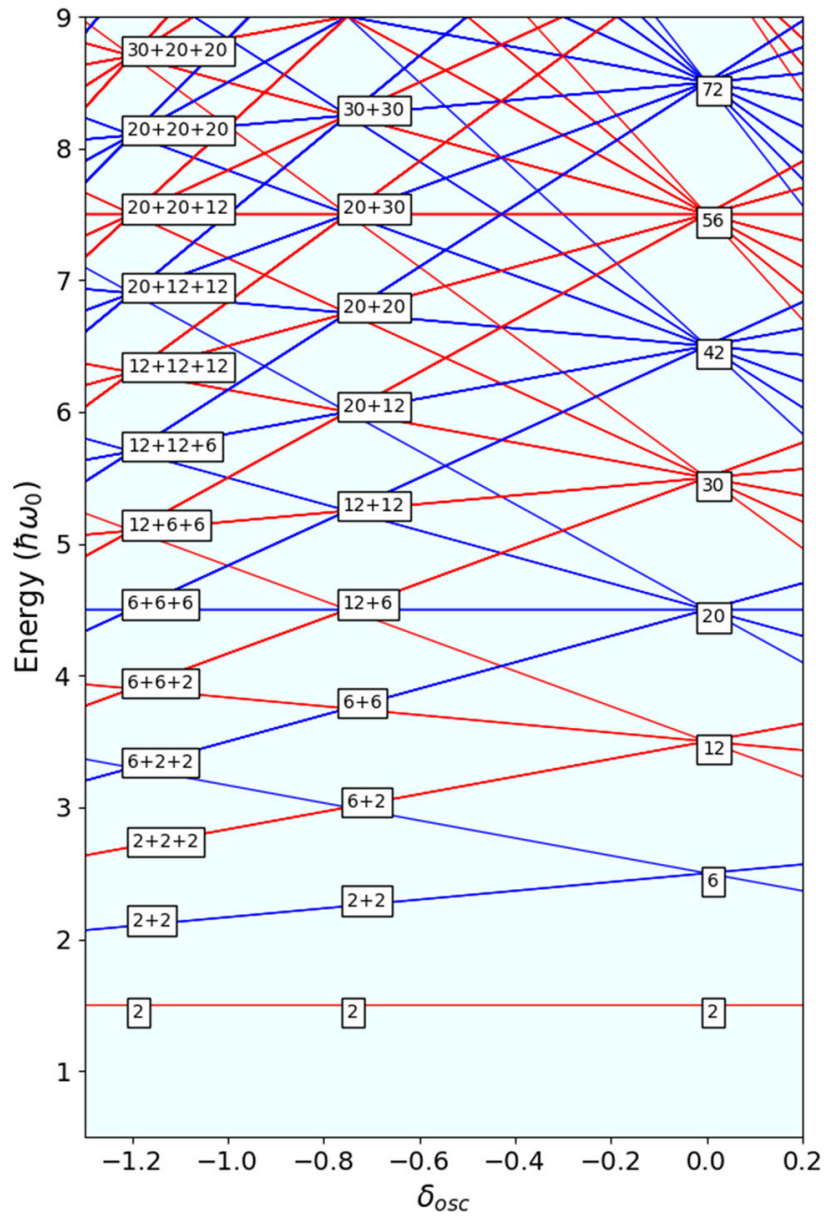

Fig. 5 The oblate side of the DHO. The numbers show the decomposition of degeneracies at 1:2 and 1:3 deformations into patterns of 2 and 3 number sequences

such structures is provided in terms of the multi-centre oscillator scheme, or Harvey-model, from which triangular nuclei may be assembled from chains of alpha-particles building sequentially larger and larger triangular arrangements.

The densities of the first four triangular nuclei are plotted in Fig. 6. These correspond to arrangements of 3, 6, 10 and 15 $\alpha$-particles, respectively. The associated harmonic oscillator configurations are:

$3:[0,0,0],[1,0,0],[0,1,0]$

$6:[0,0,0],[1,0,0],[0,1,0],[2,0,0],[0,2,0],[1,1,0](4)$

$10:[0,0,0],[1,0,0],[0,1,0],[2,0,0],[0,2,0],[1,1,0]$,

$[3,0,0],[0,3,0],[2,1,0],[1,2,0]$

$15:[0,0,0],[1,0,0],[0,1,0],[2,0,0],[0,2,0],[1,1,0]$,

$[3,0,0],[0,3,0],[2,1,0],[1,2,0],[4,0,0]$,

$[0,4,0],[3,1,0],[1,3,0],[2,2,0]$

In other words, the structures ultilise all of the orbitals available without populating any with $n_{z}=0$. These are
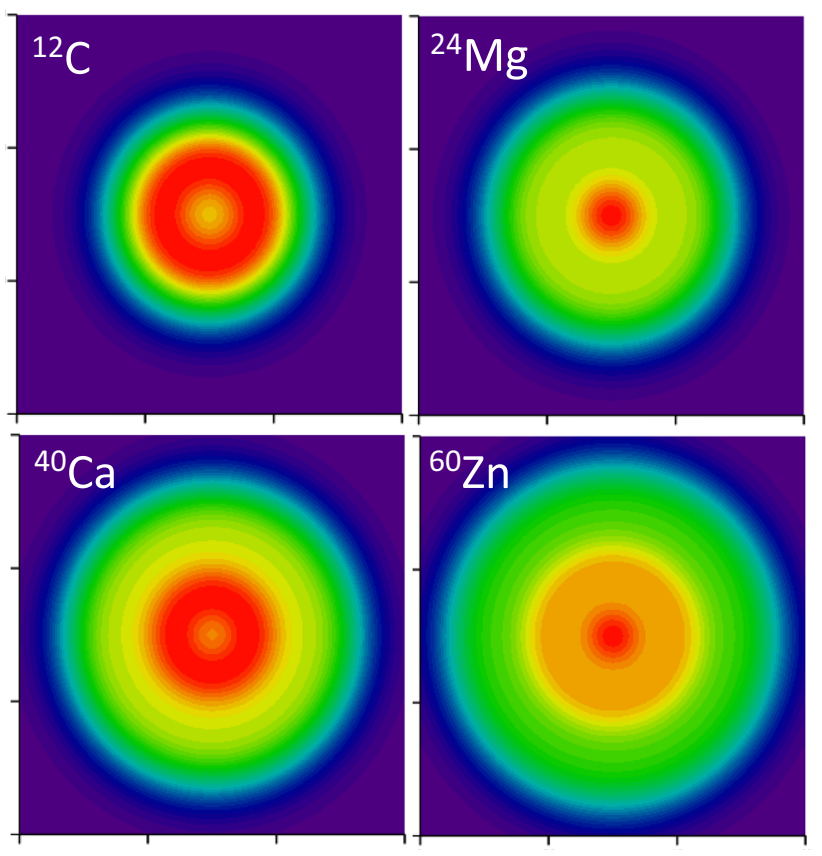

Fig. 6 The first four triangular structures as calculated in the DHO

the nuclei in Fig. 5 which are on the oblate side below the $[0,0,1]$ prolate aligned orbital which is associated with the spherical degeneracy 6 . The nuclei aside from ${ }^{12} \mathrm{C}$, where it describes the ground state, are all pretty exotic where the excitation energy would be at high energy. These 2D nuclei are also found in the calculations of the $\alpha$-cluster model when constrained to produce solutions for two-dimensional systems [12].

Due to the rotational symmetry the triangular structure cannot be observed explicitly, but one needs to extract the location of the $\alpha$-particles in order to probe the underlying structure. This can be performed either by exploiting point symmetries to transform the harmonic oscillator wavefunctions into multicentre wavefunctions, as was performed in Ref. [7], or to construct single centre wavefunctions from the multicentre $\alpha$-particle wavefunctions.

In Ref. [7] the system ${ }^{12} \mathrm{C}$ was studied and it was found that using the three oscillator levels $[0,0,0],[1,0,0]$ and $[0,1,0]$ that the three $\alpha$-particles could be projected, with their arrangement being triangular. Using the three transforms

$$
\begin{aligned}
& \phi_{1}=\frac{1}{\sqrt{3}}\left(\psi_{0}+\sqrt{\frac{3}{2}} \psi_{1}+\frac{1}{\sqrt{2}} \psi_{2}\right) \\
& \phi_{2}=\frac{1}{\sqrt{3}}\left(\psi_{0}-\sqrt{2} \psi_{2}\right) \\
& \phi_{3}=\frac{1}{\sqrt{3}}\left(\psi_{0}-\sqrt{\frac{3}{2}} \psi_{1}+\frac{1}{\sqrt{2}} \psi_{2}\right)
\end{aligned}
$$

The result is shown in Fig. 7, where the densities form the vertices of an equilateral triangle. 


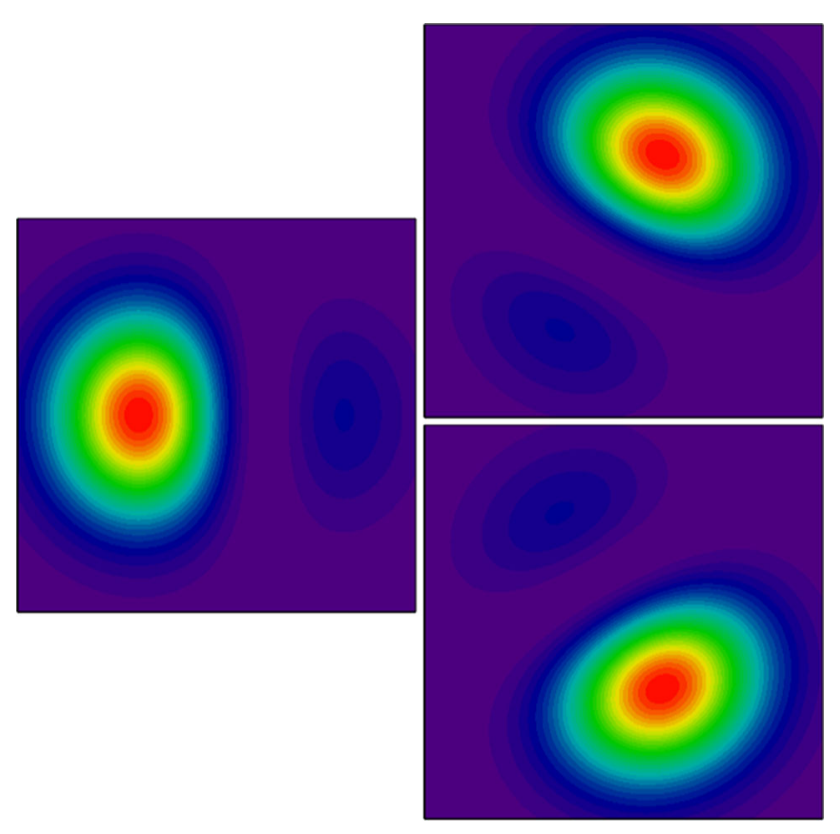

Fig. 7 The wavefunctions $\phi_{1}, \phi_{2}$ and $\phi_{3}$ corresponding to the linear combinations of $[0,0,0],[1,0,0]$ and $[0,1,0]$

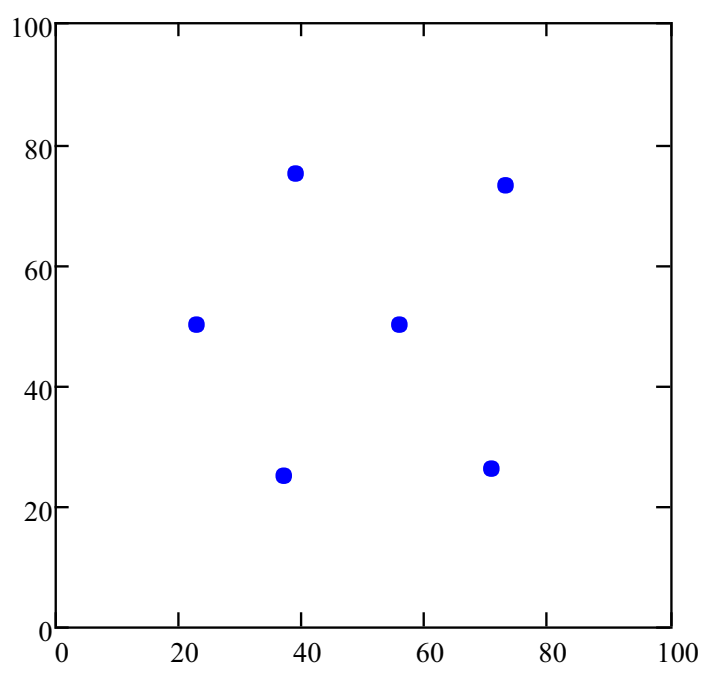

Fig. 8 The points of central density for the transformation of the harmonic oscillator wavefunctions $[0,0,0],[1,0,0],[0,1,0],[2,0,0],[0,2,0]$ and $[1,1,0]$. The horizontal and vertical scales are in arbitrary units $(\%)$ and match those for ${ }^{24} \mathrm{Mg}$ in Fig. 6

The extension of this idea, i.e. that it might be possible to transform the $3 \mathrm{HO}$ wavefunctions into their point symmetries to the six wavefunctions to their point symmetries, is illustrated in Fig. 8. Interestingly, it is not the triangle that emerges, but a collection of equilateral triangles in the form of a hexagonal pattern, as seen in the $\alpha$-cluster model calculations [12].

A possible interpretation of the degeneracies associated with Fig. 5 is given in Fig. 9.
These degeneracies patterns build the triangular numbers $3,6,10,15$ with clusters being developed at each vertex. For example, the $3 \alpha$-triangle transitions to a $3 \times{ }^{16} \mathrm{O}$ triangle or the $6 \alpha$-triangle to $6 \times{ }^{16} \mathrm{O}$. The precise connection with the structure found in Fig. 8 is yet to be completely understood. However, the hexagonal structure is the same for the 6-centred triangle and the structure shown.

\subsection{The Hoyle-state}

The oblate deformed harmonic oscillator may also be used to consider potential structures associated with the perhaps the most famous of cluster states, the Hoyle-state [14].

The Hoyle state exists at $7.65 \mathrm{MeV}$ in ${ }^{12} \mathrm{C}$ and is famed for its contribution to stellar nucleosynthesis of ${ }^{12} \mathrm{C}$ [15]. Carbon is synthesised through the triple- $\alpha$ process in which two $\alpha$-particles first combine to make ${ }^{8} \mathrm{Be}$ and before the unstable ${ }^{8} \mathrm{Be}$ decays a third $\alpha$-particle is captured, with the Hoyle-state being a gateway that enhances that process by 7-8 orders of magnitude. This is the second excited state in ${ }^{12} \mathrm{C}$ and has a pronounced $\alpha$-cluster structure.

Much work has been done to understand the nature of the structure of this state. For example, energy levels have been predicted for ${ }^{12} \mathrm{C}$ by the AMD approach [16], resonating group method (RGM) [17,18], generator coordinate method (GCM) [19-21] and Fermionic Molecular Dynamics [22]. These models demonstrate the influence of the $3 \alpha$ cluster structure. However, as yet a definitive conclusion is yet to be reached as to the precise structure, but there are a number of things that are clear. First, theoretical approaches assign it to a triangular rather than linear arrangement of the $\alpha$ particles and experimentally there are a number of states with energy and spin systematics which are consistent with this picture [15].

The ground state of ${ }^{12} \mathrm{C}$ has an oblate structure with the harmonic oscillator structure $[0,0,0],[1,0,0]$ and $[0,1,0]$. One possible excitation would be the configuration $[0,0,0],[1,0,0]$ and $[2,0,0]$, where an $\alpha$-particle is promoted to the $[2,0,0]$ orbital. This gives the linear structure shown in Fig. 2. However, this structure has been excluded from an experimental perspective, though it may appear in the monopole strength which is experimentally found in this region [23].

One therefore needs to look at alternative excitations which could be associated with the Hoyle-state. On the oblate side of deformation, the next available energy levels are $[0,0,1],[2,0,0],[0,2,0]$ and $[1,1,0]$. The most favourable configuration is the one associated with the states $[0,0,0],[1,0,0]$ and $[1,1,0]$. The equations for $\phi_{1}, \phi_{2}$ and $\phi_{3}$, above, have been used to plot the densities associated with the three-centres which are projected from the harmonic oscillator wavefunctions. What is observed is that a isosceles triangle is formed, rather than the equilateral for the ${ }^{12} \mathrm{C}$ ground state. This is 


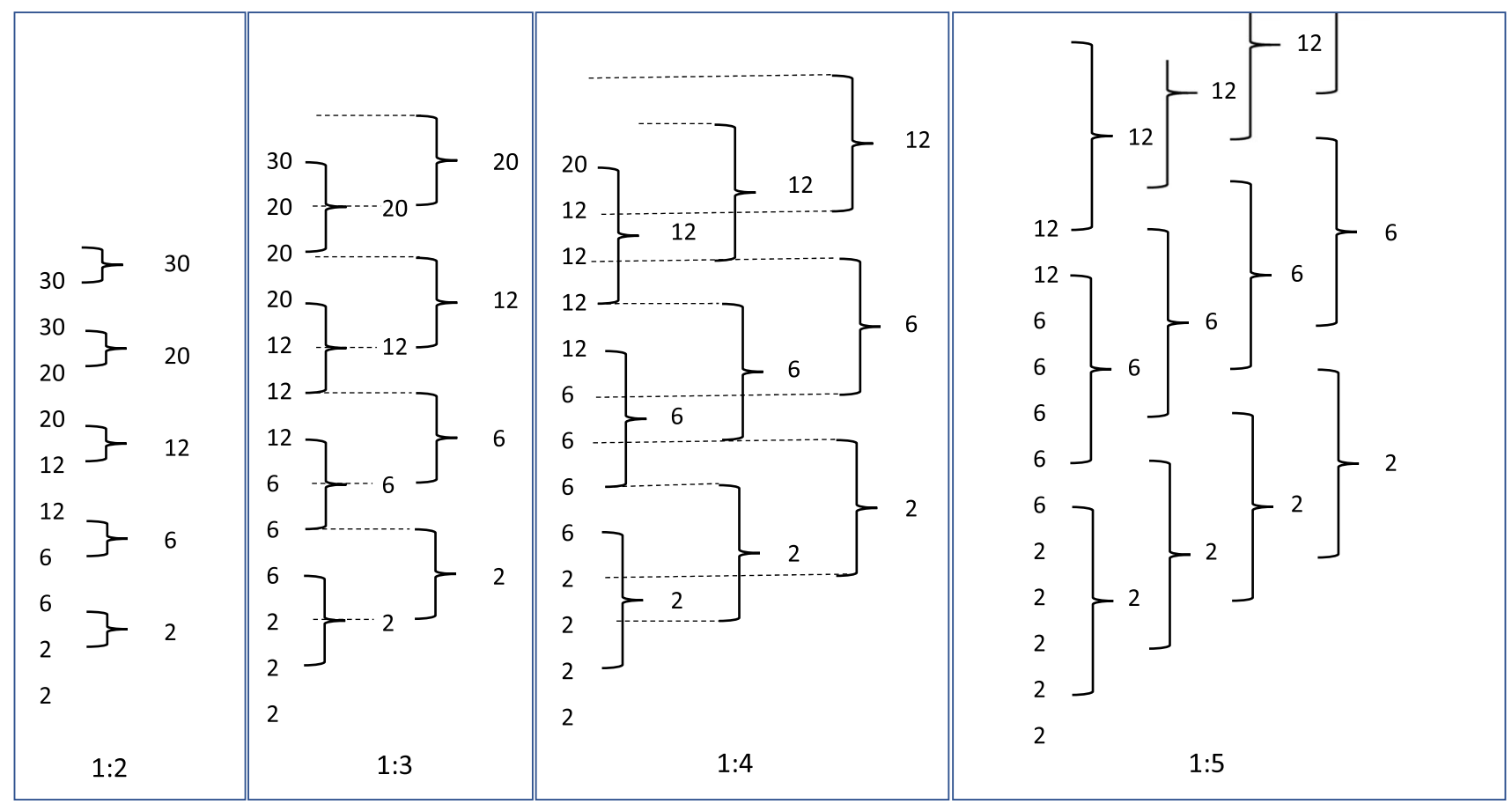

Fig. 9 The decomposition of the degeneracies at oblate deformations of 1:2, 1:3, 1:4 and 1:5
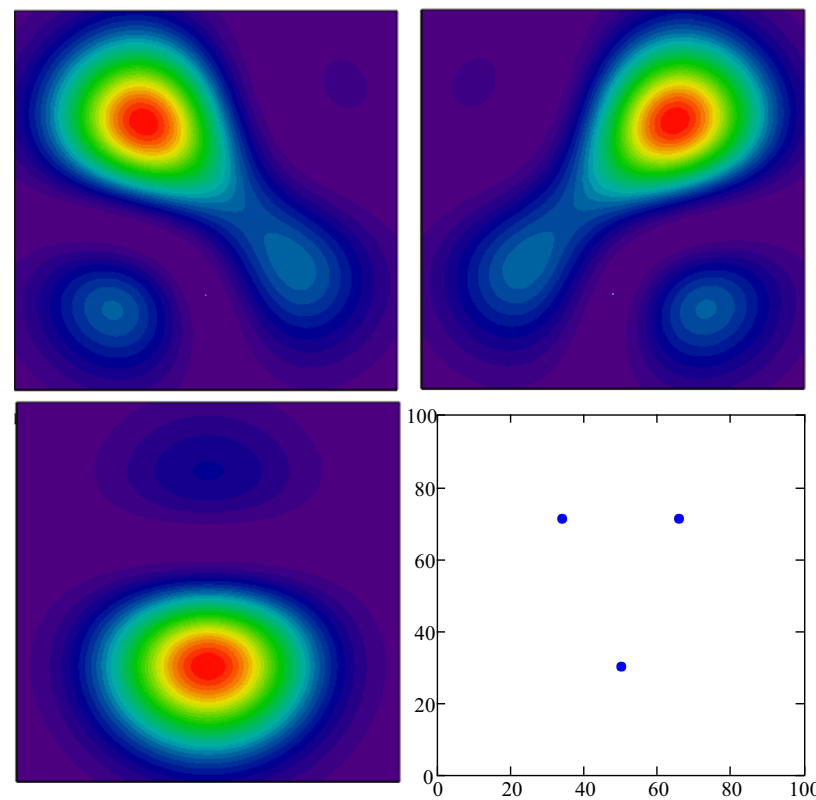

Fig. 10 The wavefunctions $\phi_{1}, \phi_{2}$ and $\phi_{3}$ corresponding to the linear combinations of $[0,0,0],[1,1,0]$ and $[0,1,0]$. The horizontal and vertical scales of the bottom right plot are in arbitrary units (\%) and match those for the other three density plots

consistent with the $a b$ initio calculations (e.g. see Ref. [1]) which reveal an ${ }^{8} \mathrm{Be}-\alpha$ type structure for the Hoyle-state.

The above observations reflect the present level of understanding, with work ongoing to provide a more comprehensive description of these oblate systems.

\section{Prolate molecular structures}

In this section we return to the topic of the description of nuclei on the prolate side of deformation. Molecular structures were predicted in the early stages of the understanding of the structure of light nuclei [24] and have been studied extensively [25-29].

It has been shown above that the prolate nuclei have welldeveloped cluster structures when the shells are closed and that these clusters are those associated with spherical nuclei such as ${ }^{4} \mathrm{He},{ }^{16} \mathrm{O}$ and ${ }^{40} \mathrm{Ca}$ as the clusters. The subset of nuclei that are $\alpha$-conjugate and have the property that each harmonic oscillator level is fully occupied, is relatively small. The question as to what happens when valence neutrons are added to such cluster systems needs therefore to be also understood.

In instances when the cluster structure is well-developed and a valence neutron (or proton) is added, it is found that the underlying clustering has a profound impact on the structure of the states. The orbitals of the valence particle are described in terms of molecular orbitals formed from the linear combination of the individual orbitals at each cluster centre $[1,2]$.

There are some simple examples of such cluster states which include the case of ${ }^{9} \mathrm{Be}$. Here the valence neutron lies in a $p$-orbital outside the $\alpha$-particle core. The linear combination of the $p$-orbitals gives rise to molecular $\pi$ and $\sigma$-bonds. These two molecular orbitals correspond to linear combinations of $p$-type ( $L=1$ ) orbitals at the two centres. The $\sigma$ molecular orbital has the densities of the dumbbell shape of the $p$-orbital aligned with the separation axis, with a projec- 

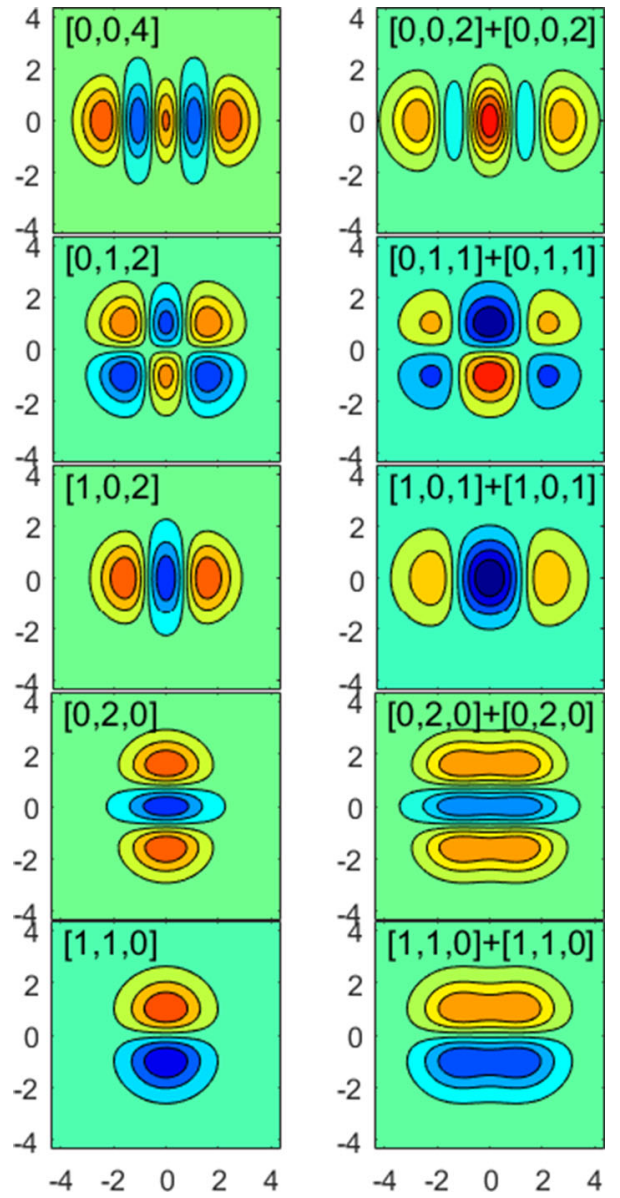

Fig. 11 Comparison between 5 of the 6 possible molecular orbitals for the valence neutron in ${ }^{33} \mathrm{~S}$ calculated in the DHO (left) and the multicentre, Hückle, method (right). The [2,0,0] orbital has not been shown. The patterns in the wave-function amplitudes match, demonstrating that the DHO valence orbitals all have a molecular multi-centre nature. The orbitals are labelled by their harmonic oscillator quantum numbers $\left[n_{x}, n_{y}, n_{z}\right]$

tion of the angular momentum of the molecular orbital onto that axis of zero. The $\pi$-orbital has the dumbbell densities perpendicular to the separation and with an orbital angular momentum projection of $1 \hbar$. These delocalised orbitals result in the binding of the two $\alpha$-particles, which are unbound in ${ }^{8} \mathrm{Be}$. In this instance, the molecular orbitals can be traced to collection of orbitals with a degeneracy of 6 in Fig. 1 which lie immediately above the two levels labelled with degeneracy 2 at a deformation of $2: 1\left(\delta_{O S C}=0.6\right)$. These latter two levels would be those occupied by an ${ }^{8} \mathrm{Be}$ nucleus in the ground state. In other words, the molecular orbitals associated with ${ }^{9} \mathrm{Be}$ are the next available orbitals once the two $\alpha$ cluster system has been formed and hence would be associated with low-lying states in ${ }^{9} \mathrm{Be}$, as observed experimentally [2].

A recent study has examined the nature of all of the orbitals above the closure of a shell related to a cluster structure [8].
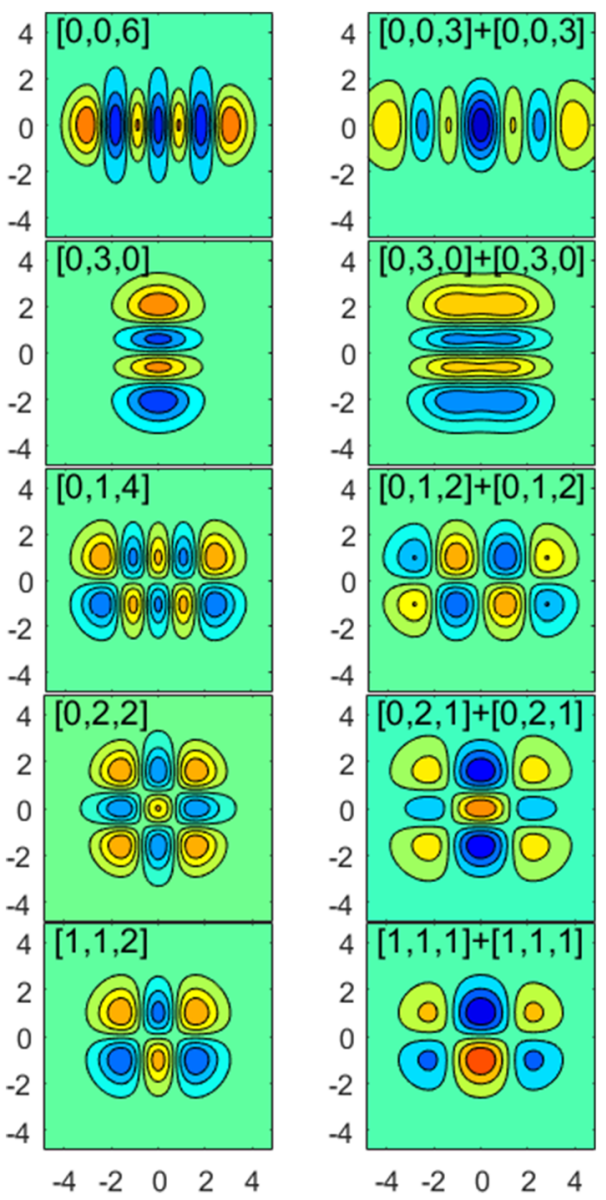

Fig. 12 Comparison between selected molecular orbitals ( 5 of the 10 available possibilities) for the valence neutron in ${ }^{81} \mathrm{Zr}$ calculated in the DHO (left) and the multi-centre, Hückle, method (right). The patterns in the wave-function amplitudes match, demonstrating that the DHO valence orbitals have a molecular multi-centre nature. The orbitals are labelled by their harmonic oscillator quantum numbers $\left[n_{x}, n_{y}, n_{z}\right]$

Figures 11 and 12 show two cases. These correspond to building the molecular orbitals around the cluster structures ${ }^{16} \mathrm{O}+{ }^{16} \mathrm{O}$ and ${ }^{40} \mathrm{Ca}+{ }^{40} \mathrm{Ca}$, i.e. ${ }^{33} \mathrm{~S}$ and ${ }^{81} \mathrm{Zr}$.

${ }^{33} \mathrm{~S}$ can be considered as a $2: 1$ deformed nucleus with two ${ }^{16} \mathrm{O}$ clusters with a delocalised neutron. In the DHO model, where the oscillation frequency in the $z$-direction is half that in the $x$ or $y$ directions, the orbitals occupied linked to ${ }^{32} \mathrm{~S}$ are $\left[n_{x}, n_{y}, n_{z}\right]=[0,0,0],[0,0,1],[0,0,2],[0,1,0]$, $[1,0,0],[0,0,3],[0,1,1]$ and $[1,0,1]$. The orbitals available, associated with the next shell closure, for the valence neutron are $[0,0,4],[0,1,2],[1,0,2],[0,2,0],[2,0,0]$ and $[1,1,0]$, these are all degenerate. In the two-centre description, each ${ }^{16} \mathrm{O}$ cluster occupies the orbitals $[0,0,0],[1,0,0],[0,1,0]$ and $[0,0,1]$, which leaves the following orbitals available for the valence neutron centred on each cluster $\left[n_{x}, n_{y}, n_{z}\right]=$ $[0,0,2],[0,2,0],[2,0,0],[0,1,1],[1,0,1]$ and $[1,1,0]$. Linear combinations of these latter orbitals give rise to those in the DHO in a 1 to 1 match. 
Given that the neutron could exist at either cluster centre with equal probability, the resulting two-centre molecular wave-functions can be matched to the DHO orbitals with a one-to-one correspondence:

$$
\begin{aligned}
& {[0,0,4] } \equiv \frac{1}{\sqrt{2}}[[0,0,2]+[0,0,2]], \\
& {[0,1,2] \equiv \frac{1}{\sqrt{2}}[[0,1,1]+[0,1,1]], } \\
& {[1,0,2] \equiv \frac{1}{\sqrt{2}}[[1,0,1]+[1,0,1]], } \\
& {[0,2,0] \equiv \frac{1}{\sqrt{2}}[[0,2,0]+[0,2,0]], } \\
& {[2,0,0] \equiv \frac{1}{\sqrt{2}}[[2,0,0]+[2,0,0]], } \\
& {[1,1,0] \equiv \frac{1}{\sqrt{2}}[[1,1,0]+[1,1,0]] . }
\end{aligned}
$$

Here the $n_{z}$ quantum number in the DHO is the sum of the two-centre $n_{z}$ quantum numbers and the $n_{x}$ and $n_{y}$ values remain unchanged as predicted by the two-centre shell model. In this instance the orbitals in the DHO immediately above those responsible for the ${ }^{32} \mathrm{~S}$ cluster structure all have a molecular structure.

Similarly, ${ }^{81} \mathrm{Zr}$, can be thought of as two ${ }^{40} \mathrm{Ca}$ clusters with a single delocalised neutron. In the 2:1 DHO model, the orbitals filled with nucleons from the ${ }^{80} \mathrm{Zr}$ core are $\left[n_{x}, n_{y}, n_{z}\right]=[0,0,0],[0,0,1],[0,0,2],[0,1,0],[1,0,0]$, $[0,0,3],[0,1,1],[1,0,1],[0,0,4],[0,1,2],[1,0,2],[0,2,0]$, $[2,0,0],[1,1,0],[0,0,5],[0,1,3],[1,0,3],[0,2,1],[2,0,1]$ and $[1,1,1]$. The next highest energy orbitals available for the delocalised neutron are then $[0,0,6],[0,1,4],[1,0,4],[1,1,2]$, $[0,2,2],[2,0,2],[0,3,0],[3,0,0],[1,2,0]$ and $[2,1,0]$. These are all degenerate at the 2:1 deformation. In the two-centre approach, each ${ }^{40} \mathrm{Ca}$ cluster occupies the orbitals: $[0,0,0]$, $[1,0,0],[0,1,0],[0,0,1],[2,0,0],[0,2,0],[0,0,2],[1,1,0]$, $[1,0,1]$ and $[0,1,1]$. The valence neutron then occupies the orbitals $[0,0,3],[0,3,0],[3,0,0],[1,1,2],[1,0,2],[1,1,1]$, $[0,2,1],[2,0,1],[1,2,0]$ and $[2,1,0]$ around each centre. Again the combination of the single-centre orbitals give the complete set of valence DHO orbitals. Once more this is a demonstration that the DHO orbitals have a molecular character.

As the delocalised neutron in ${ }^{81} \mathrm{Zr}$ exists at a much higher energy than ${ }^{33} \mathrm{~S}$, the degeneracy is much larger. This means that there are a greater number of molecular orbitals which must be constructed using linear combinations, but there is still only one possible combination of wave-functions from each cluster centre which give the correct orbital shape, Fig. 12. Once again, it can be seen there is a good match between the properties of the 2:1 deformed orbitals and the explicit two-centre molecular counterparts. These two examples demonstrate that at a deformation of 2:1 the deformed harmonic oscillator orbitals above the deformed shell clo- sure all have a molecular structure and thus the deformed harmonic oscillator explicitly includes the 2-centre molecular behaviour.

This feature evident at a deformation of 2:1 has been explored at other deformations and asymmetric choices of cluster and the same conclusions are reached [8].

The conclusions of this analysis are pretty clear. Above every shell-closure for the prolate nuclei lies a series of energy levels which correspond to the molecular orbitals which would have been formed from the linear combination of the valence orbitals around each spherical cluster component. In other words, irrespective of the cluster structure, symmetric or asymmetric, there exist valence orbitals which would allow the covalent exchange of protons or neutrons between the clusters. This makes the molecular symmetries a ubiquitous feature of prolate deformed nuclei.

Of course, it is one thing for such symmetries to exist and it is another for them to survive the complexity which arises from the mixing of the sea of other possible structures and states, as illustrated in Fig. 4.

\section{Conclusion}

The deformed harmonic oscillator has long been the basis for calculations of nuclear properties and underlies the energy level schemes predicted by the Nilsson deformed shell model which are the basis for understanding of many nuclear properties. The appearance of shell-structure in the Nilsson scheme can be traced to shell-like structures in the deformed harmonic oscillator. Moreover, the predictions of the DHO map to those of more complex models which dispense with the mean-field and deal with nucleon-nucleon interactions, increasingly grown from QCD degrees of freedom. The present contribution has explored the breadth of symmetries associated with the DHO in terms of the cluster structures that emerge for prolate shell-gaps, the molecular structures which occur for the valence, covalent, orbitals built around the clusters and some of the emergent thinking related to oblate nuclei. There remains work to be done, but it is clear that queryPlease provide an explanation as to why there is no data or why the data will not be deposited. Your explanation will be displayed as 'Authors' comment'.the symmetries that exist are a crucial element to understanding the structure of nuclei and not just light nuclei.

Acknowledgements The ideas outlined here were presented in the workshop organised by Mahir Hussein to commemorate the sad passing of Paulo Gomes in Sao Paulo, Brazil. It is some how fitting for them to be documented at the passing of Mahir. Mahir Hussein was a man of great warmth, depth and intelligence. His contributions to the field of reactions of light and often exotic nuclei have been significant and together with Paulo, central to establishing the Sao Paulo group as a force within the field of nuclear physics. I remember fondly the many 
discussions with Mahir and his encouragement. Greatness is not just measured in the accomplishment of the individual but the encouragement and facilitation of the success of others. Mahir measured well.

Funding University of Birmingham

Data Availability Statement This manuscript has no associated data or the data will not be deposited. [Authors' comment: This is a theoretical study and no experimental data.]

Open Access This article is licensed under a Creative Commons Attribution 4.0 International License, which permits use, sharing, adaptation, distribution and reproduction in any medium or format, as long as you give appropriate credit to the original author(s) and the source, provide a link to the Creative Commons licence, and indicate if changes were made. The images or other third party material in this article are included in the article's Creative Commons licence, unless indicated otherwise in a credit line to the material. If material is not included in the article's Creative Commons licence and your intended use is not permitted by statutory regulation or exceeds the permitted use, you will need to obtain permission directly from the copyright holder. To view a copy of this licence, visit http://creativecomm ons.org/licenses/by/4.0/.

\section{References}

1. M. Freer, H. Horiuchi, Y. Kanada-En'yo, D. Lee, U.-G. Meißner, Rev. Mod. Phys. 90, 035004 (2018)

2. M. Freer, Rep. Progress Phys. 70, 2149 (2007)

3. Clusters in Nuclei, Volumes 1 to 3, Ed. C. Beck, Springer

4. A. Bohr, B.R. Mottelson, Nuclear Structure, vol. 2 (W. A. Benjamin, New York, 1975)

5. W. Nazarewicz, J. Dobaczewski, Phys. Rev. Letts. 68, 154 (1992)
6. W.D.M. Rae, Fifth Int. Conf. Clustering Aspects in Nucl. and Subnucl. Systems, Kyoto 1988, J. Phys. Soc. Jpn 58 (1989), Suppl. p77

7. M. Freer, R.R. Betts, A.H. Wuosmaa, Nucl. Phys. A 587, 36 (1995)

8. R. Canavan and M. Freer 2020 J. Phys. G 47095102 (2020)

9. S. Bailey et al., Phys. Rev. C 100, 051302(R) (2019)

10. K. Ikeda, H. Takigawa, H. Horiuchi, Prog. Theor. Phys. Suppl. Extra Number 464 (1968)

11. K.A. Erb, D.A. Bromley, Treatise Heavy-Ion Sci. 3, 201 (1975)

12. J. Zhang, W.D.M. Rae, Nucl. Phys. A 564, 252 (1993)

13. W.D.M. Rae, J. Zhang, Mod. Phys. Lett. A 09, 599 (1994)

14. F. Hoyle, Astrophys. J. Suppl. Ser. 1, 12 (1954)

15. M. Freer, H.O.U. Fynbo, Progress Particle Nucl. Phys. 78, 1 (2014)

16. Y. Kanada, En'yo Prog. Theor. Phys. 117, 655 (2007)

17. T. Marumori, J. Phys. Soc. Jpn. 44, 225 (1978)

18. M. Kamimura, Nucl. Phys. A 351, 456 (1981)

19. E. Uegaki, S. Okabe, Y. Abe, H. Tanaka, Prog. Theor. Phys. 57, $1262(1977)$

20. E. Uegaki, Y. Abe, S. Okabe, H. Tanaka, Prog. Theor. Phys. 59, 1031 (1978)

21. E. Uegaki, Y. Abe, S. Okabe, H. Tanaka, Prog. Theor. Phys. 62 , 1621 (1979)

22. M. Chernykh et al., Phys. Rev. Letts. 98, 032501 (2007)

23. K.C.W. Li, arXiv preprint arXiv:2011.10112 (2020)

24. L.R. Hafstad, E. Teller, Phys. Rev. 54, 681 (1938)

25. M. Seya, M. Kohno, S. Nagata, Prog. Theor. Phys. 65, 204 (2000)

26. W. von Oertzen, Z. Physik A 354, 37 (1996)

27. W. von Oertzen, Z. Physik A, 357, 355, (1997); also Il Nuovo Cimento A 110, 895, (1997)

28. N. Itagaki, S. Okabe, Phys. Rev. C 61, 044306 (2000)

29. M. Kimura, H. Horiuchi, Phys. Rev. C 69, 051304 (2004) 